\title{
Low-lying spectrum for lattice Dirac operators with twisted mass
}

\section{Christof Gattringer*}

Institut für Physik, FB Theoretische Physik

Karl-Franzens-Universität Graz

A-8010 Graz, Austria.

E-mail: christof.gattringereuni-graz.at

\section{Stefan Solbrig}

Institut für Theoretische Physik

Universität Regensburg

D-93040 Regensburg, Germany.

E-mail: stefan.solbrigephysik.uni-r.de

\begin{abstract}
We analyze the low-lying spectrum and eigenmodes of lattice Dirac operators with a twisted mass term. The twist term expels the eigenvalues from a strip in the complex plane and all eigenmodes obtain a non-vanishing matrix element with $\gamma_{5}$. For a twisted Ginsparg-Wilson operator the spectrum is located on two arcs in the complex plane. Modes due to non-trivial topological charge of the underlying gauge field have their eigenvalues at the edges of these arcs and obey a remnant index theorem. For configurations in the confined phase we find that the twist mainly affects the zero modes, while the bulk of the spectrum is essentially unchanged.
\end{abstract}

XXIIIrd International Symposium on Lattice Field Theory

25-30 July 2005

Trinity College, Dublin, Ireland

${ }^{*}$ Speaker. 
In the last few years lattice QCD formulations with a twisted mass term were studied in great detail [1]-[5] and the latest results were reviewed at this conference [6]. The interest of the lattice community in twisted mass QCD is based on the fact that the twisted mass term protects the Dirac operator from small eigenvalues, thus avoiding exceptional configurations. This allows one to work at small quark masses and the results can be extrapolated to vanishing twist.

However, the fact that no small eigenvalues appear implies that also zero modes of the Dirac operator are excluded. Zero modes of the Dirac operator in turn are, via the index theorem [7], related to infrared structures of the gauge field carrying topological charge. This connection already raises the question we address in this contribution: How does topological charge of the gauge field manifest itself in the eigensystem of the Dirac operator with a twisted mass term?

We stress that this question is not only of academic interest, since topological objects are believed to play an important role in the QCD vacuum. Thus it should be understood how topological objects interact with the fermionic degrees of freedom in the presence of a twisted mass term.

For two mass-degenerate flavors of fermions, $u$ and $d$, the twisted mass Dirac operator $\mathscr{D}$ has the form

$$
\mathscr{D}=\left(D_{0}+m\right) \mathbb{1}_{2}+i \mu \gamma_{5} \tau_{3} \quad, \quad \tau_{3}=\operatorname{diag}(1,-1),
$$

where $\mathbb{1}_{2}$ and $\tau_{3}$ act in flavor space. For our analysis it is convenient to analyze the Dirac operators for $u$ and $d$ separately by setting $D_{u}=D(\mu)$ and $D_{d}=D(-\mu)$ with

$$
D(\mu)=D_{0}+m+i \mu \gamma_{5}
$$

The Dirac operator $D_{0}$ is the 1-flavor Dirac operator, discretized on the lattice without doublers. A common choice is Wilson's Dirac operator, but here we will also consider (for theoretical purposes) a Dirac operator that is chiral, i.e., obeys the Ginsparg-Wilson relation [8].

A property which we assume for the operator $D_{0}$, and which is obeyed by Wilson's Dirac operator and also the overlap operator, is $\gamma_{5}$-hermiticity,

$$
\gamma_{5} D_{0} \gamma_{5}=D_{0}^{\dagger}
$$

For the twisted operator $D(\mu)$ this property implies the generalized $\gamma_{5}$-hermiticity relation [9]

$$
\gamma_{5} D(\mu) \gamma_{5}=D(-\mu)^{\dagger} .
$$

The generalized $\gamma_{5}$-hermiticity relation can be explored to derive results for the eigensystem of the Dirac operator. We denote an eigenvector of $D(\mu)$ with eigenvalue $\lambda$ by $\psi_{\lambda}$ and find for its matrix element with $\gamma_{5}$ :

$$
\begin{aligned}
& \lambda\left(\psi_{\lambda}, \gamma_{5} \psi_{\lambda}\right)=\left(\psi_{\lambda}, \gamma_{5} D(\mu) \psi_{\lambda}\right)=\left(\psi_{\lambda}, D(-\mu)^{\dagger} \gamma_{5} \psi_{\lambda}\right)=\left(D(-\mu) \psi_{\lambda}, \gamma_{5} \psi_{\lambda}\right)= \\
& \left(\left[D(\mu)-i 2 \mu \gamma_{5}\right] \psi_{\lambda}, \gamma_{5} \psi_{\lambda}\right)=\lambda^{*}\left(\psi_{\lambda}, \gamma_{5} \psi_{\lambda}\right)+i 2 \mu\left(\psi_{\lambda}, \psi_{\lambda}\right)=\lambda^{*}\left(\psi_{\lambda}, \gamma_{5} \psi_{\lambda}\right)+i 2 \mu
\end{aligned}
$$

We have used (3) and the fact that the eigenvectors are normalized to 1. From the last equation follows

$$
\left(\psi_{\lambda}, \gamma_{5} \psi_{\lambda}\right)=\frac{\mu}{\operatorname{Im} \lambda}
$$



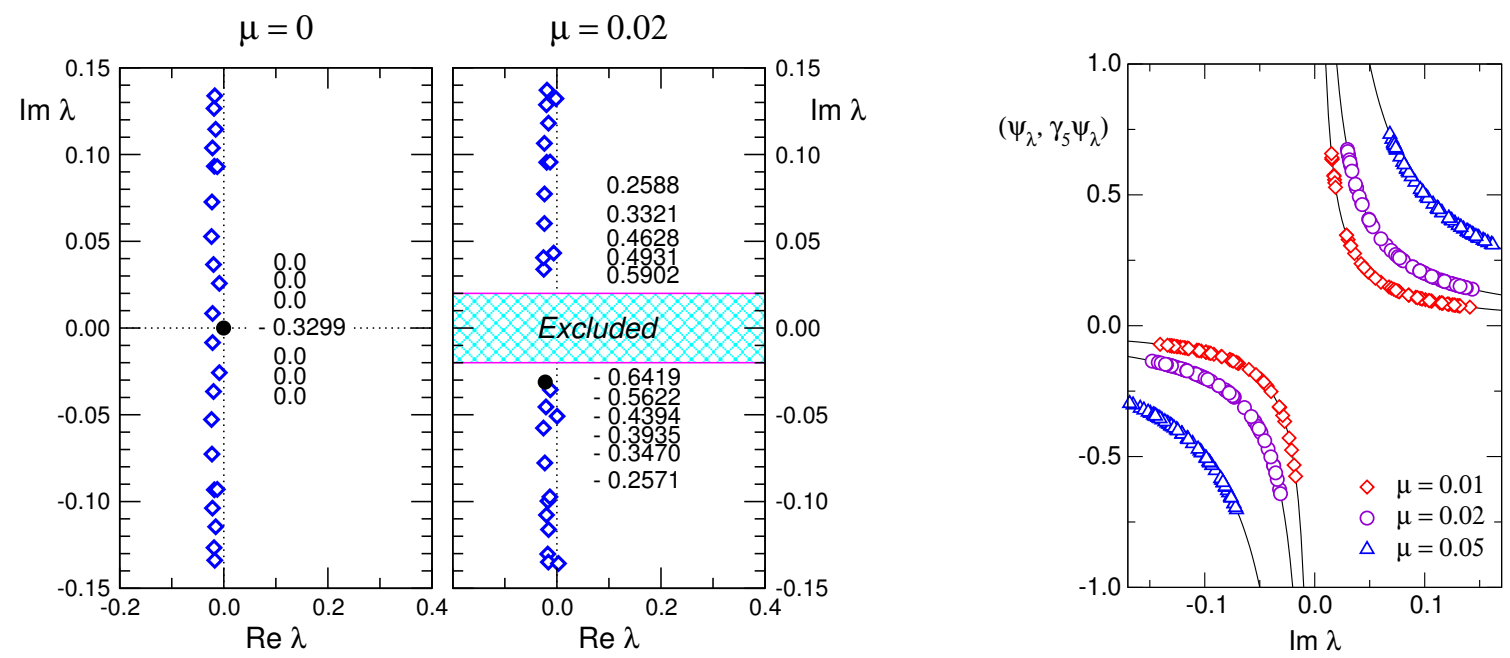

Figure 1: Left-hand side plot: Spectra of the Dirac operator in the complex plane for $\mu=0.0$ and $\mu=$ 0.02. The numbers next to the symbols for the eigenvalues are the matrix elements of the corresponding eigenvectors with $\gamma_{5}$. Right-hand-side plot: $\gamma_{5}$ matrix elements of the eigenvectors $\psi_{\lambda}$ as a function of $\operatorname{Im} \lambda$. The full curves are the hyperbolas of Eq. (5).

This formula implies that all eigenvectors have non-vanishing chirality which decreases monotonically with $\operatorname{Im} \lambda$. This is very different from the case without twist term, where only eigenmodes with real eigenvalue have a non-vanishing matrix element with $\gamma_{5}$. These modes correspond to zero modes in the continuum and are of topological nature.

Using the fact that $\gamma_{5}$ is bounded, i.e., $\left|\left(\psi_{\lambda}, \gamma_{5} \psi_{\lambda}\right)\right| \leq 1$, we find $(\mu \geq 0)$

$$
|\operatorname{Im} \lambda| \geq \mu \text {. }
$$

Thus all eigenvalues have an imaginary part larger or equal the twist parameter $\mu$ (positive in our convention). The twist term expels the spectrum from a strip of width $2 \mu$ along the real axis and thus the smallest eigenvalues have a modulus with a size of at least $\mu$.

The basic properties of the spectrum and the eigenmodes are illustrated in Figure 1 which was generated using the twisted Wilson Dirac operator on a quenched gauge field configuration of topological charge $Q=+1$ on a lattice of size $16^{4}$ at a lattice spacing of $a=0.094 \mathrm{fm}$ as determined from the Sommer parameter [10].

In the left-hand side plot we compare the spectra in the complex plane for vanishing twist and at a twist of $\mu=0.02$ in lattice units. As the twist term is turned on, the eigenvalues are shifted away from the real axis. The topological eigenvalue (filled symbol) is shifted in the negative imaginary direction. It is obvious that the spectrum is no longer symmetric with respect to reflection at the real axis. We remark that the fluctuations of the eigenvalues in horizontal direction are not reduced by the twist term. The numbers next to the symbols representing the eigenvalues $\lambda$ are the values for the matrix elements $\left(\psi_{\lambda}, \gamma_{5} \psi_{\lambda}\right)$ of the corresponding eigenvectors $\psi_{\lambda}$.

In the right-hand side plot of Figure 1 we show these matrix elements as a function of $\operatorname{Im} \lambda$ for three different values of $\mu$. The symbols represent the numerical data, the full curves show the hyperbolas of Eq. (5). 

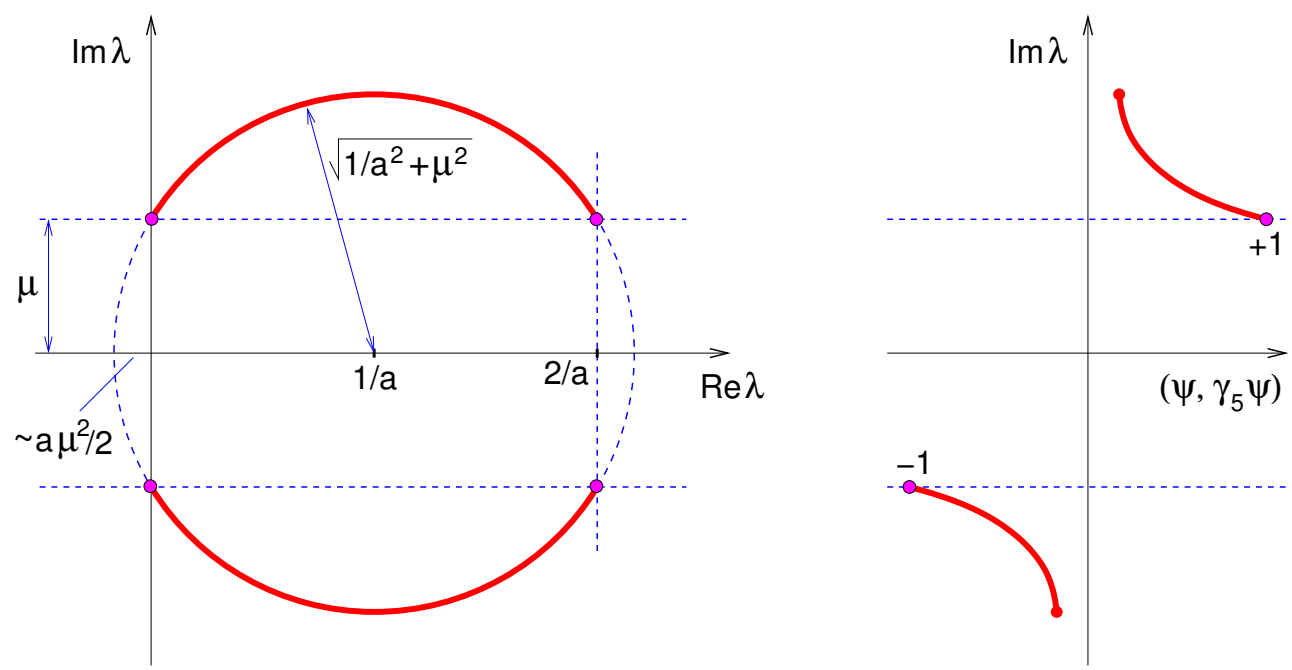

Figure 2: Schematic representation of our results for spectrum (left-hand side plot) and the matrix element with $\gamma_{5}$ (right-hand side plot) for a chiral Dirac operator with twisted mass term.

As discussed, the focus of this paper is on the low lying spectrum of the twisted mass lattice Dirac operator and the role of the index theorem. For the index theorem it is important to use a setting where chiral symmetry is properly implemented on the lattice and to consider the role of the twisted mass term in this case. Thus we require now the Dirac operator $D_{0}$ (before the twist term is turned on) to obey the Ginsparg-Wilson relation,

$$
\gamma_{5} D_{0}+D_{0} \gamma_{5}=a D_{0} \gamma_{5} D_{0},
$$

which governs chiral symmetry on the lattice. In this case $D_{0}$ can have exact zero modes $\psi_{0}^{ \pm}$which are chiral, i.e., $\gamma_{5} \psi_{0}^{ \pm}= \pm \psi_{0}^{ \pm}$. They are related to the topological charge $Q$ of the gauge field via the index theorem,

$$
Q=n_{-}-n_{+},
$$

where $n_{+}$and $n_{-}$are the numbers of right- and left-handed zero modes [11].

Since the zero modes $\psi_{0}^{ \pm}$of $D_{0}$ are chiral, they are also eigenmodes of $D(\mu)$ with eigenvalue $\pm i \mu$, with a plus sign for the right-handed mode and a minus sign for a left-handed zero mode. Thus in the presence of the twisted mass term we can identify a remnant of the index theorem which still has the form of Eq. (8), but $n_{-}$and $n_{+}$are now interpreted as the number of eigenvalues $+i \mu$ and $-i \mu$.

From (1) one finds $D_{0}=D(\mu)-i \mu \gamma_{5}$ and inserting this expression into (7) one obtains after a few lines of algebra (use (3) and $D(-\mu)=D(\mu)-i 2 \mu \gamma_{5}$ ),

$$
D(\mu)+D(\mu)^{\dagger}=a D(\mu) D(\mu)^{\dagger}-a \mu^{2} .
$$

Sandwiching this equation between eigenvectors $\psi_{\lambda}$ gives rise to the equation

$$
\lambda+\lambda^{*}=a \lambda \lambda^{*}-a \mu^{2},
$$




$$
Q=-1 \text {, real Polyakov loop }
$$

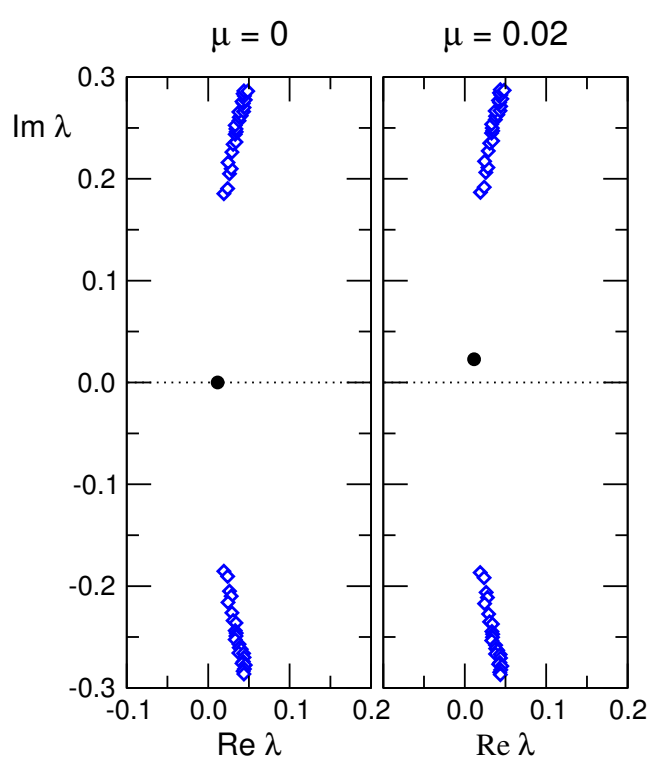

$\mathrm{Q}=+1$, complex Polyakov loop

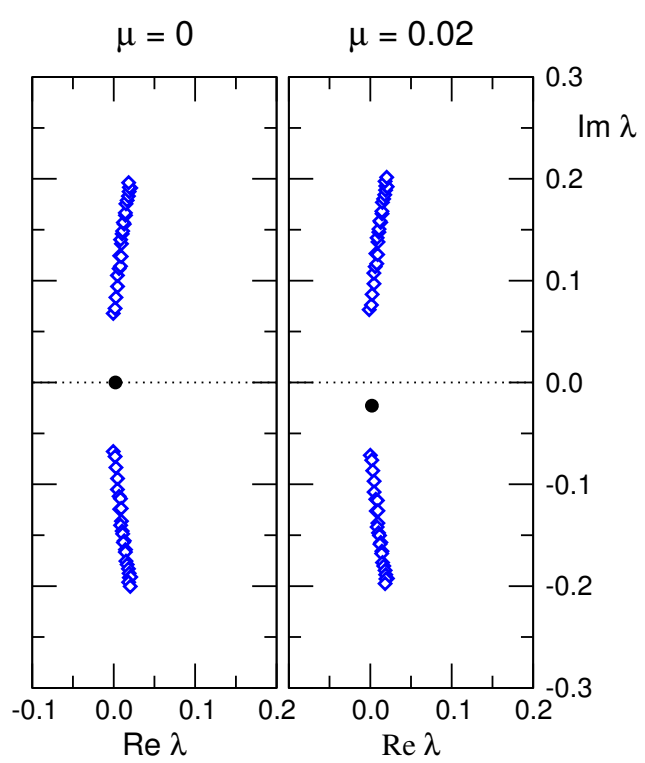

Figure 3: Spectrum of the Dirac operator in the deconfined, chirally symmetric, phase. We compare the untwisted case to the situation at $\mu=0.02$. The left-hand side plot is for a configuration with $Q=-1$ and real Polyakov loop, while the right-hand side plot is for $Q=1$ and complex Polyakov loop.

for the corresponding eigenvalue $\lambda$. Setting $\lambda=x+i y$ one finds that this is the equation for a circle in the complex plane. This "stretched Ginsparg-Wilson circle" has center $1 / a$ and radius $\sqrt{1 / a^{2}+\mu^{2}}$. Since $|\operatorname{Im} \lambda| \geq \mu$ we find that the spectrum of $D(\mu)$ is confined to the two arcs shown in the left-hand side plot of Fig. 2. The topological eigenmodes with eigenvalues $\pm i \mu$ fall one the edges of the two arcs (on the other edges their doubler partners are found). The matrix elements of the eigenvectors with $\gamma_{5}$ still obey Eq. (5). The right-hand side plot of Fig. 2 illustrates the results for the $\gamma_{5}$-matrix element.

Our discussion of a Ginsparg-Wilson operator with a twisted mass term shows that the zero modes of a chiral Dirac operator without twist, $D_{0}$, are protected by chiral symmetry and remain unchanged as the twist term is turned on (only the eigenvalue changes). Instead, when using the Wilson Dirac operator for $D_{0}$, this protection is only approximate. In [5] characteristic properties of the topological zero mode (edge mode), such as its localization and $\gamma_{5}$ matrix element, were studied for different values of $\mu$. While for the twisted Wilson operator these properties changed considerably, for the approximately chiral CI operator [12] they remained essentially unchanged.

Another question that was addressed in [5] is the role of the twisted mass term in the deconfined (chirally symmetric) phase. In the chirally symmetric phase the spectrum of the Dirac operator has a gap near the origin (up to isolated zero modes) which implies, via the Banks-Casher relation [13], a vanishing chiral condensate. Turning on the twisted mass term mainly affects the zero modes. As seen in Fig. 3, they are shifted up or down, depending on their chirality. The size of the spectral gap remains essentially unchanged. 


\section{References}

[1] R. Frezzotti, P. A. Grassi, S. Sint, and P. Weisz [ALPHA collaboration], Lattice QCD with a chirally twisted mass term, JHEP 0108 (2001) 058 [hep-lat/ 0101001 ]; R. Frezzotti, S. Sint, and P. Weisz [ALPHA collaboration], O(a) improved twisted mass lattice QCD, JHEP 0107 (2001) 048 [hep-lat/ 0104014 ]; M. Della Morte, R. Frezzotti, J. Heitger, and S. Sint [ALPHA collaboration], Cutoff effects in twisted mass lattice QCD, JHEP 0110 (2001) 041 [hep-lat / 0108019 ].

[2] R. Frezzotti and G. C. Rossi, Chirally improving Wilson fermions. I: O(a) improvement, JHEP 0408 (2004) 007 [hep-lat/ 0306014 ]; R. Frezzotti and G. C. Rossi, Chirally improving Wilson fermions. II: Four-quark operators, JHEP 0410 (2004) 070 [hep-l at / 04070 02];

[3] F. Farchioni et al., Twisted mass quarks and the phase structure of lattice QCD, Eur. Phys. J. C 39 (2005) 421 [hep-lat / 0406039 ]; G. Münster, On the phase structure of twisted mass lattice QCD, JHEP 0409 (2004) 035 [hep-lat/ 040700 6]; S. R. Sharpe and J. M. S. Wu, The phase diagram of twisted mass lattice QCD, Phys. Rev. D 70 (2004) 094029 [hep-lat / 0407025 ].

[4] K. Jansen, A. Shindler, C. Urbach and I. Wetzorke [XLF Collaboration], Scaling test for Wilson twisted mass QCD, Phys. Lett. B 586 (2004) 432 [hep-lat/0312013]; W. Bietenholz et al. [XLF Collaboration], Going chiral: Overlap versus twisted mass fermions, JHEP 0412 (2004) 044 [hep-lat/0411001].

[5] C. Gattringer and S. Solbrig, Remnant index theorem and low-lying eigenmodes for twisted mass fermions, Phys. Lett. B 621 (2005) 195 [hep-lat/ 0503004 ].

[6] A. Shindler, Plenary talk at this conference.

[7] M. F. Atiyah and I. M. Singer, The Index Of Elliptic Operators. 5, Annals Math. 93 (1971) 139.

[8] P. H. Ginsparg and K. G. Wilson, A remnant of chiral symmetry on the lattice, Phys. Rev. D 25 (1982) 2649.

[9] S. Aoki and A. Gocksch, Spontaneous breaking of parity in quenched lattice QCD with Wilson fermions, Phys. Lett. B 231 (1989) 449.

[10] C. Gattringer, R. Hoffmann, and S. Schaefer, Setting the scale for the Lüscher-Weisz action, Phys. Rev. D 65 (2002) 094503 [hep-lat/ 0112024 ].

[11] P. Hasenfratz, V. Laliena, and F. Niedermayer, The index theorem in QCD with a finite cut-off, Phys. Lett. B 427 (1998) 125 [hep-lat/9801021].

[12] C. Gattringer, A new approach to Ginsparg-Wilson fermions, Phys. Rev. D 63 (2001) 114501 [hep-lat/ 0003005$]$; C. Gattringer, I. Hip, and C. B. Lang, Approximate Ginsparg-Wilson fermions: A first test, Nucl. Phys. B 597 (2001) 451 [hep-lat / 0007042 ].

[13] T. Banks and A. Casher, Chiral symmetry breaking in confining theories, Nucl. Phys. B 169 (1980) 103. 